\title{
Biological activities of essential oils from the genus Ferula (Apiaceae)
}

\author{
Amirhossein Sahebkar, Mehrdad Iranshahi \\ Biotechnology Research Center and School of Pharmacy, Mashhad University of Medical Sciences \\ (MUMS), Mashhad, Iran
}

\begin{abstract}
The genus Ferula (Apiaceae) comprises about 170 species occurring from central Asia westward to northern Africa. This genus is well-known in folk medicine for the treatment of various organ disorders. Most of Ferula species possess strong aromatic smell that is due to the presence of essential oil or oleoresin in their different organs. This article reviews anti-bacterial, anti-fungal and other biological activities of Ferula oils reported to date. For medicinal applications, the chemical composition of volatile oils obtained from different Ferula species is summarized in Appendix.
\end{abstract}

Keywords: Apiaceae, essential oil, Ferula

The Apiaceae or Umbelliferae is a family of usually aromatic plants with hollow stems commonly known as umbellifers. This family is well represented in the Iranian flora, at least with 112 genera, 316 species, and 75 endemic species [1]. Notable members of this family include Anethum graveolens (Dill), Anthriscus cerefolium (chervil), Angelica spp. (Angelica), Apium gravolence (celery), Carum carvi (caraway), Coriandrum sativum (coriander), Cuminum cyminum (cumin), Foeniculum vulgare (fennel), Ferula gummosa (galbanum), and Pimpinella anisum (anise). The aromatic smell of most species is due to the presence of essential oil or oleoresin in their different organs [2]. Pictures of Ferula species are shown in Fig. 1.

The genus Ferula, belonging to the family Apiaceae, comprises about 170 species. These are produced from central Asia westward to northern Africa [3]. The Iranian flora comprises of 30 species of Ferula, of which some are endemic $[4,5]$. The popular Persian name of the most species is "Koma" [5].

The chemistry of this genus has been studied by many investigators. To date, more than 70 species of

Correspondence to: Dr. Mehrdad Iranshahi, Vakilabad Boulevard, School of Pharmacy, Mashhad University of Medical Sciences, Mashhad 91775-1365, Iran. E-mail: Iranshahim @mums.ac.ir
Ferula have been investigated chemically [6-8]. The plants of this genus are well documented as a good source of biologically active compounds such as derivatives [9-17], and sulfur containing compounds [18-24].

Several species of this genus have been used in traditional medicine for the treatment of various organ disorders. Among different Ferula species that have been used as natural remedies, F. assa-foetida (used as anticonvulsant, carminative, antispasmodic, diuretic, aphrodisiac, antihelmintic, tonic, and laxative, alterative, etc.), F. badrakema and F. gummosa (both used as anti-convulsant, tonic, anti-hysteric, decongestant, treatment of neurological disorders, and stomachache), and F. persica (used as laxative, carminative, antihysteric, treatment of lumbago, diabetes, rheumatism, and backache) are most famous [25-28].

Recent investigations have led to the discovery of some new biological activities of the plants of this genus. These include anti-microbial, anti-fungal, antinociceptive, anti-inflammatory, anti-convulsant, antioxidant, anti-mycobacterial, anti-spasmodic, and hypotensive activities [29-39]. At least, part of the biological activities of the plants of this genus can be attributed to their essential oils [29-31].

Essential oils are the subtle, highly concentrated, aromatic, and volatile liquids. These are extracted from the flowers, seeds, leaves, stems, bark, and roots of 

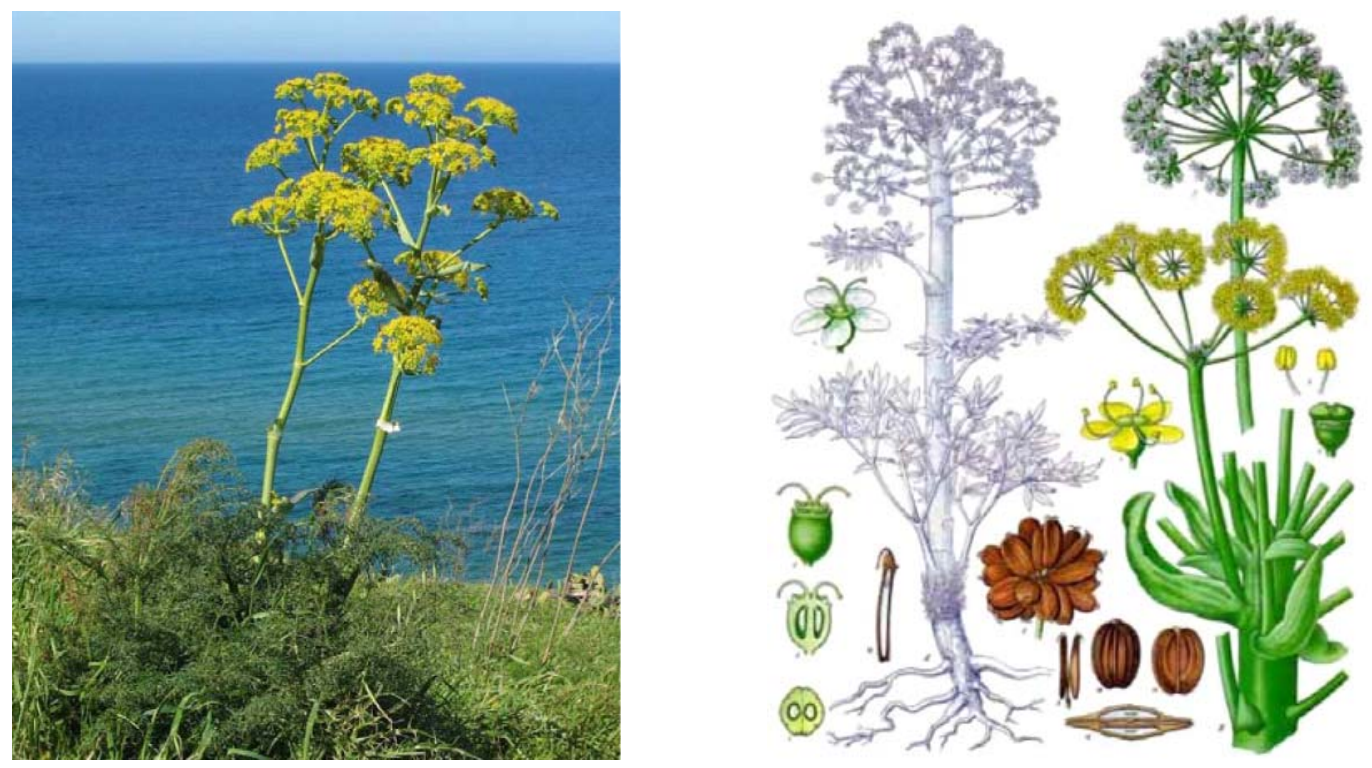

Fig. 1 Natural and schematic pictures of Ferula species.

plants, usually through steam or hydro-distillation. These natural oils are mixtures of complex and volatile compounds that are synthesized by aromatic plants as secondary metabolites. The importance of essential oils is not only confined to their natural protecting role for the host plants, but also to the fact that these oils contain properties many times more powerful than dried herbs. Among these properties are the antibacterial, anti-microbial, anti-viral, and anti-fungal activities, together with some particular medicinal effects that make essential oils a very important consideration [29-31, 40-43]. Regarding the strong odor of many Ferula species and reported effects of some isolated volatile components, it seems that essential oils of this genus have an important role in the observed biological effects of these plants.

In this article, we review biological activities of Ferula oils where stress is put on anti-microbial and anti-fungal activities of Ferula oils. Considering a recent trend to natural products including essential oils for medicinal applications, we summarize the chemical composition of volatile oils obtained from different Ferula species in Appendix.

\section{Antimicrobial activities}

In a previous survey, the essential oil from the fruits of $F$. badrakema was found to be moderately active against Staphylococcus aureus and Bacillus cereus as Gram-positive bacteria, and Candida albicans as fungal strain. However, Gram-negative bacteria (Escherichia coli and Pseudomonas aeruginosa) appeared not to be susceptible to inhibitory effects of this essential oil [29]. In another study, the essential oils from F. glauca were evaluated for antibacterial and antifungal activity. The results showed that the Gram-positive $B$. subtilis was the most sensitive strain. The essential oils also showed moderate inhibitory activity against Strptococcus mutans, Enterococcus faecalis and E. coli whereas no remarkable activity was observed against $S$. aureus and the yeast $C$. albicans (which was the most resistant strain). Findings also demonstrated that leaves and fruits essential oils were the most active oils of the plant on the tested microorganisms [30]. Concerning the F. latisecta, the polysuphide-rich fruit oil of this plant was shown to possess antibacterial activity against Gram-positive (B.cereus and in particular S. aureus) but not Gram-negative bacteria (P. aeruginosa and E.coli) and a relatively potent inhibitory activity against $C$. albicans [44]. Moreover, this oil was tested for its antifungal activity against a range of human pathogenic dermatophytes (Trichophyton mentagrophytes, T. rubrum, T. verrucosom, Microsporum canis and M. gypseum). The results showed that the oil was active against all tested dermatophytes with the most significant activity against T. rubrum and T. verrucosom [31]. In another study, the essential oil from the aerial parts of $F$. latisecta was reported to exert high inhibitory activity against the Gram-positives B. subtilis and E. faecalis, 
moderate activity against $S$. aureus, E. coli and Klebsiella pneumoniae and was inactive against $P$. aeruginosa [45]. Concerning the $F$. gummosa, it was found that the essential oil from the fruits of the plant possesses strong antibacterial and antifungal activities against Gram-positive ( $S$. aureus, $S$. epidermis and B. subtilis) and negative (E. coli, Salmonella typhi and Pseudomonas aeruginosa) bacteria and fungi (C. albicans and C. kefyr) [46]. In another study, F. gummosa seed oil was also reported to be active against Gram-positive bacteria (S. aureus, B. subtilis and E. faecalis) and E. coli. However, unlike the former study, little antibacterial activity was found from this oil against $P$. aeruginosa [47]. For F. szowitsiana, the leaf oil of the plant possessed antimicrobial activity against two strains of Gram-positive bacteria (methicillin-resistant $S$. aureus and S. epidermidis), four strains of Gramnegative bacteria (E. coli, P. aeruginosa, Proteus vulgaris and Salmonella typhimurium) and a yeast (Candida albicans). The minimal inhibitory concentration (MIC) values of the leaf oil towards the selected human pathogenic bacteria and the fungus were determined as $0.156-1.25 \mu \mathrm{g} / \mathrm{mL}$. Noteworthy, the strong antibacterial activity of this oil against methicillin-resistant $S$. aureus (MRSA, MIC $=0.156$ $\mu \mathrm{m} / \mathrm{mL}$ ) was an interesting finding [48]. In another investigation on the essential oil obtained from the aerial parts of this plant, $B$. subtilis was found to be the most sensitive strain compared with the $S$. aureus, Gram-negative bacteria (E. coli, P. aeruginosa) and fungal strains (Aspergillus niger and C. albicans) for which weaker activities of the oil were observed [49]. Finally, there is a previous report indicating the antifungal activity of $F$. assa-foetida seed oil against five species of the food borne mold Aspergillus (A. awamori, A. niger, A. flavus, A. foetidus and A. oryzae). The oil was shown to inhibit all the three stages of asexual reproduction of Aspergillus species i.e., spore germination, mycelial growth, and spore formation [50]. Findings on the antimicrobial activities of Ferula oils have been summarized in Table 1.

\section{Miscellaneous activities}

Together with the anti-microbial activities, a few miscellaneous activities have also been reported from essential oils of Ferula species. Concerning the F. gummosa, the fruit oil of the plant was evaluated for anticonvulsant activity against experimental seizures. The essential oil had no effect against seizures induced by maximal electroshock but protected mice against pentylenetetrazole-induced tonic seizures. However, the protective dose produced neurotoxicity and was too close to the $\mathrm{LD}_{50}$ of the essential oil [51]. In another investigation, the oleo-gum-resin oil of F. gummosa was reported to possess relaxant effect on rat-isolated ileum against contractions induced by $\mathrm{KCl}$ and acetylcholine. The authors stated that at least part of this inhibitory effect might be due to the $\alpha$-pinene and $\beta$-pinene components of the oil [52]. In connection with $F$. harmonis, it was revealed that seed oil of the plant could enhance erectile function in rats. However, it was reported that this oil could also cause certain toxicities if it is used for a long period and even may become a male contraceptive agent [53]. Finally, the essential oil from $F$. orientalis aerial parts was found to possess antioxidative potential in 2'-diphenyl-1picrylhydrazyl radical (DPPH) as well as $\beta$-carotene/ linoleic acid assay, though it was not as strong as that of positive control (BHT) [32].

\section{Concluding remarks}

There are only few reports on the biological activity of essential oils from Ferula species, of which the majority have investigated the anti-microbial activity of these oils. Previous findings indicate the antimicrobial activity of Ferula essential oils and their potential application as natural aromatic anti-bacterial and anti-fungal agents. The bacteriostatic and fungistatic properties of these essential oils may be associated to the high content of $\alpha$-pinene and $\beta$-pinene or polysulfides that are present in these oils and for which strong anti-microbial activities have been reported previously [54-56].

Asian countries have used herbs and minerals as nutrients, and medicine for a long time. However, their analytical studies have not been completed yet $[59,60]$. Some reports indicate side effects of traditional medicine to acknowledgement of the value of the long heritage in traditional medicine. Further analysis and understanding of herbal and mineral medicine will increase to current medical practice.

The authors have no conflict of interest to report. 


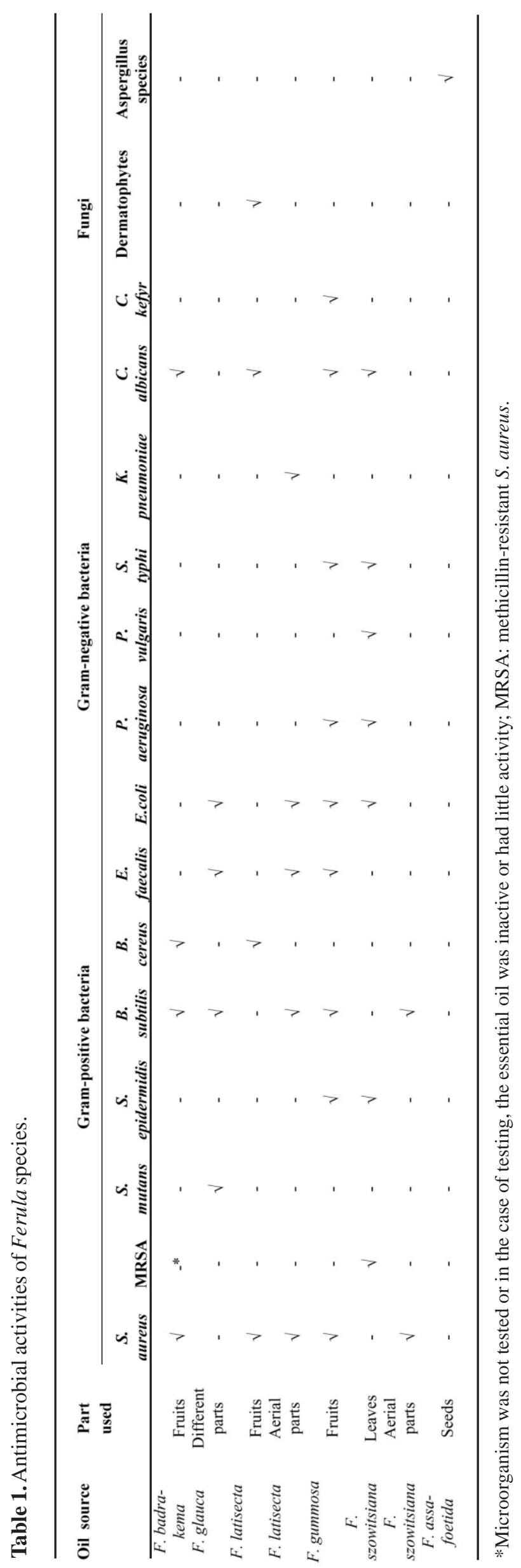




\section{Appendix}

The chemical composition of volatile oils obtained from different Ferula

Different Ferula species share some similarities in their volatile components, but there are many compositional differences. We can classify their essential oils based on their compositional differences.

The most prominent measure that can be applied to categorize Ferula oils is the presence of sulfurcontaining compounds. The essential oils obtained from F. assa-foetida, F. fukanensis, F. latisecta, $F$. persica and F. sinkiangensis contained sulfur compounds. On the other hand, other oils were devoid of these compounds among their identified components. sec-Butyl-(Z)-propenyl disulfide and secbutyl-(E)-propenyl disulfide were found to be the most prevalent sulfur-containing compounds in the essential oils of some Ferula species. The terpenoid compounds were almost the most abundant components of Ferula oils. The most frequent terpenoid compounds that occurred as main components in the essential oils were $\alpha$-pinene, $\beta$-pinene, myrcene and limonene (among monoterpene hydrocarbons); linalool, -terpineol and neryl acetate (among oxygenated monoterpenes); $\beta$-caryophyllene, germacrene $\mathrm{B}$, germacrene $\mathrm{D}$ and $\delta$-cadinene (among sesquiterpene hydrocarbons) and caryophyllene oxide, $\alpha$-cadinol, guaiol and spathulenol (among oxygenated sesquiterpenes). Despite the existing reports and considering the total number of identified Ferula species (more than 170), there are still many species uninvestigated. Therefore, conducting future studies on the chemical composition and particularly biological activities of uninvestigated Ferula oils is greatly recommended. The tabulated overviews of chemical components of Ferula species essential oils, together with the structures of the main components, are shown in Fig. 2-7.

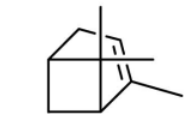

$\alpha$-Pinene

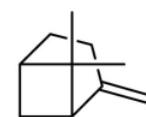

$\beta$-Pinene

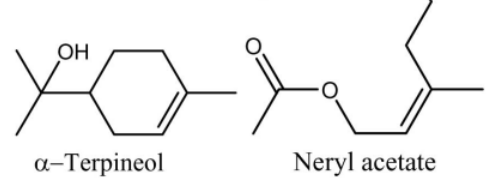

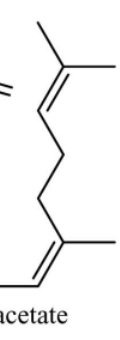
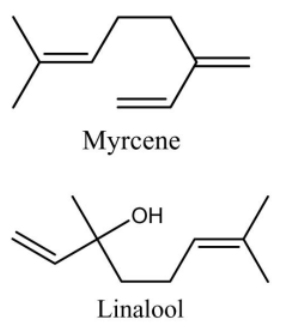

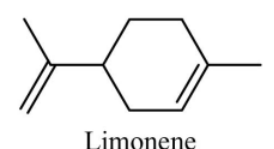

Limonene

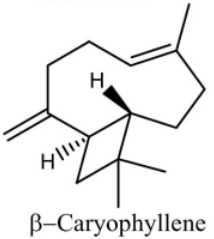<smiles>CC1=CC(=C(C)C)CCC(C)=CCC1</smiles><smiles>C=C1C=CC(CCC(C)=CCC)CCC1C(C)C</smiles><smiles>CC1=C[C@@H]2C(=C(C)CC1)CC[C@H](C(C)C)[C@H]2C(C)C</smiles>

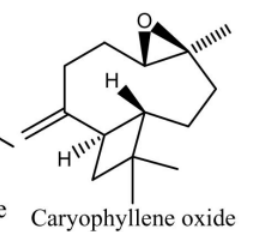<smiles>CC1=C[C@H]2[C@@H](C(C)C)CC[C@H](O)[C@]2(C)CC1</smiles><smiles>C[C@H]1CCC2=C(C[C@@H](C(C)(C)O)CC2)[C@@H]1C</smiles>

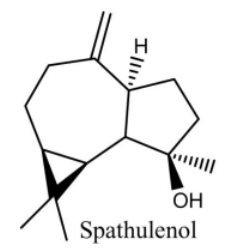

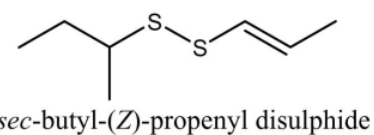

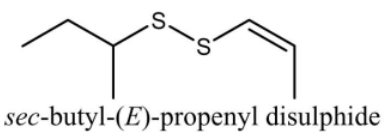

Fig. 2 Chemical structure of the most frequent main components present in the essential oils of Ferula species. 


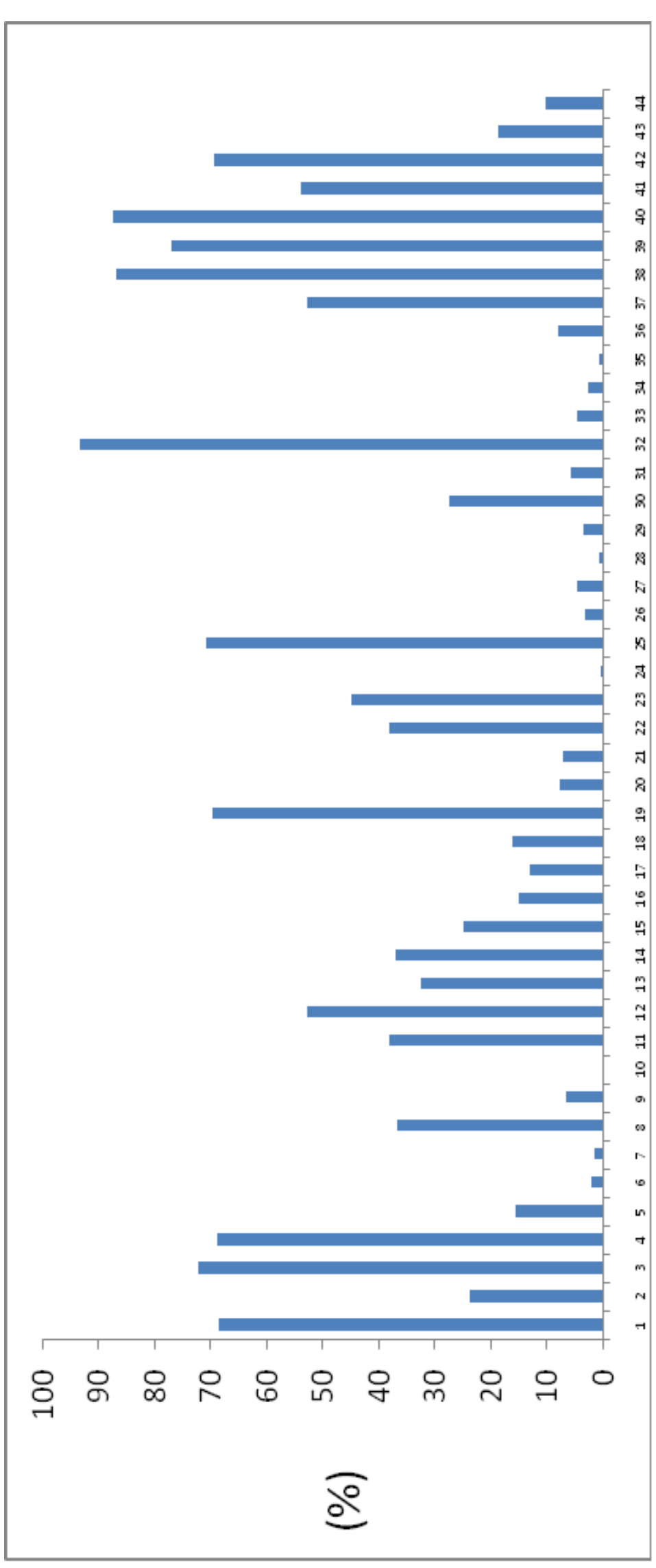

山ั

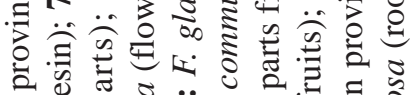

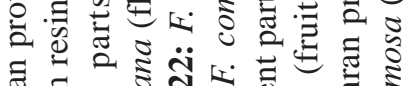

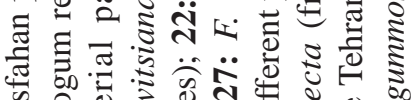

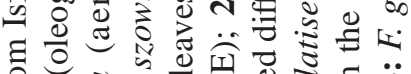

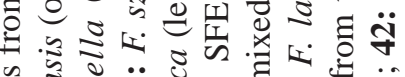

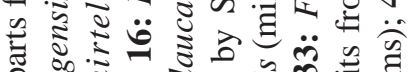

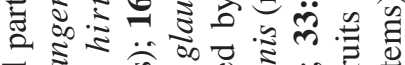

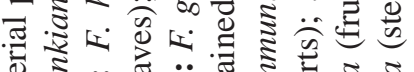
离

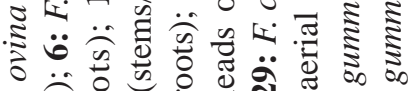

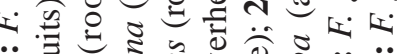

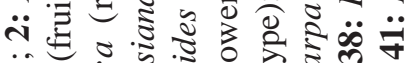

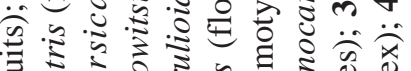

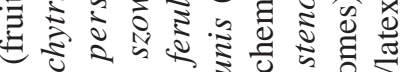

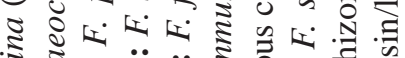
ठั

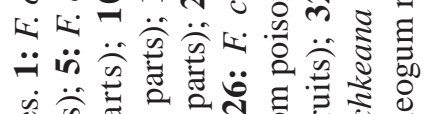

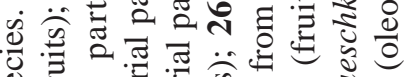

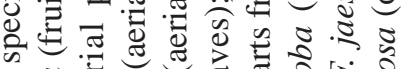
क व ป 过完

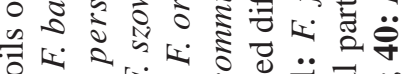
б도

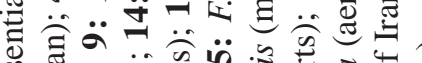

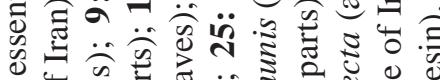
Ð

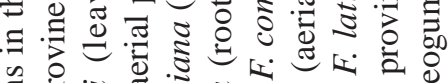

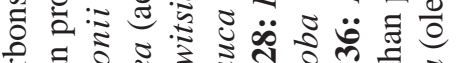

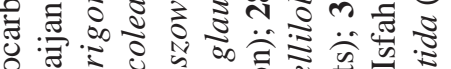

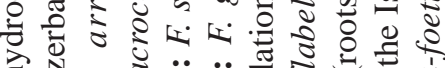

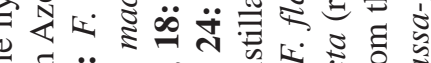

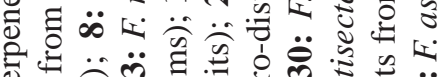

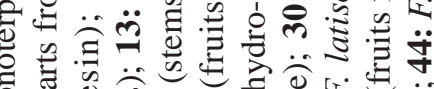

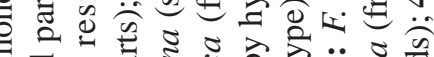

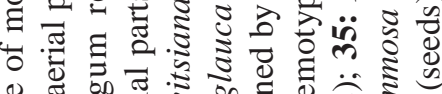

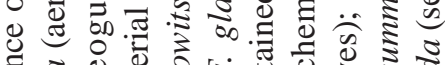

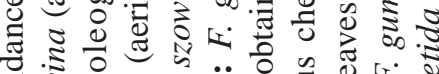
व व

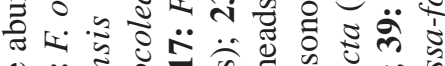

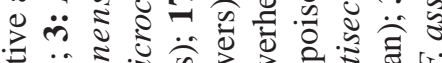

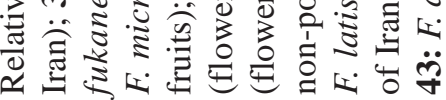
m 


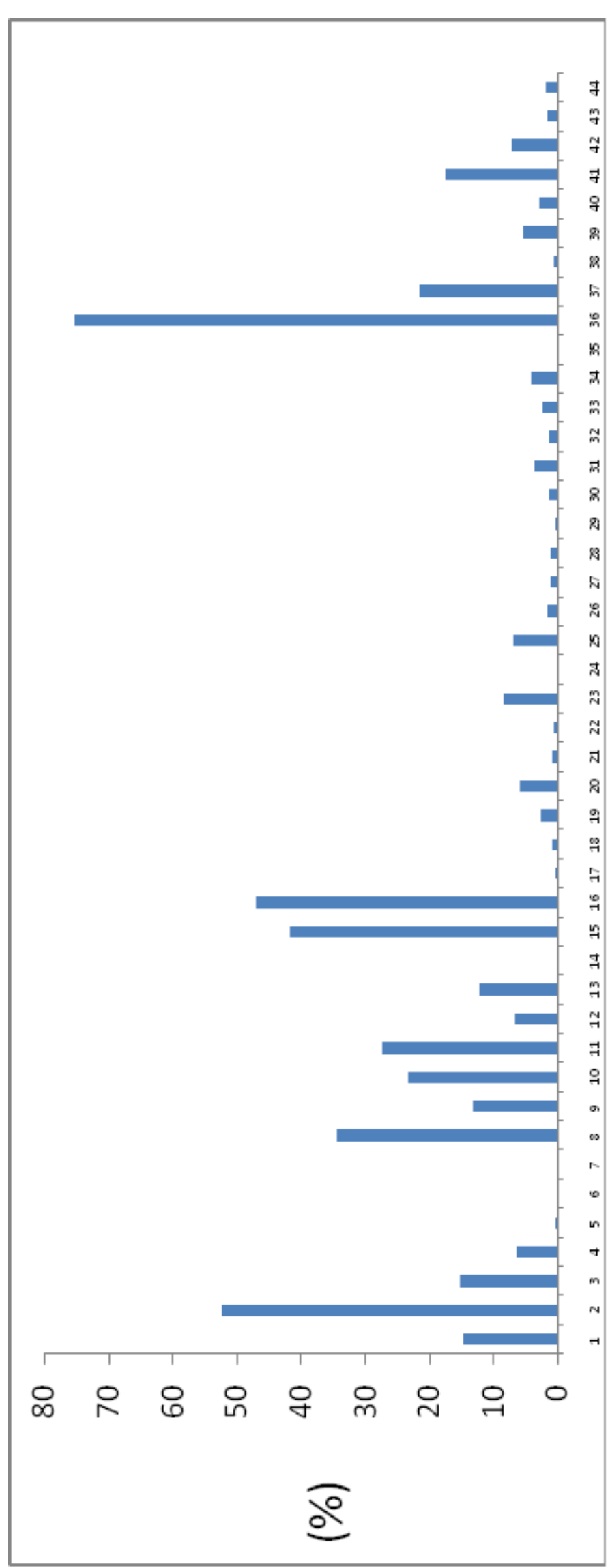

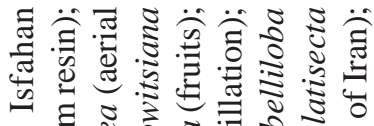

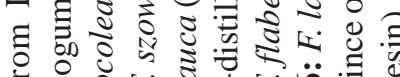

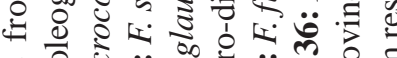

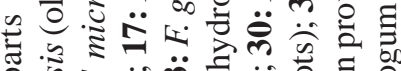

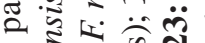

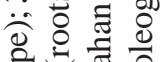

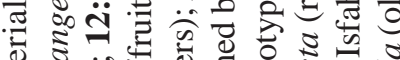

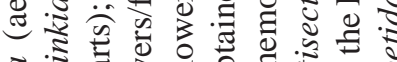
ㅇำ

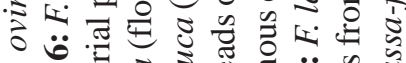

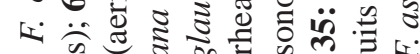

i

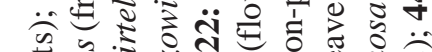

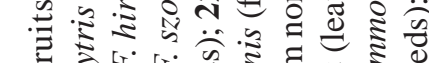

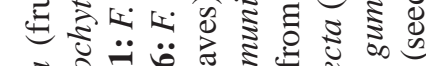

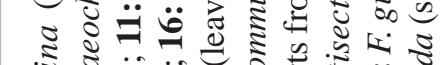

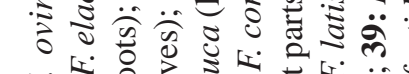

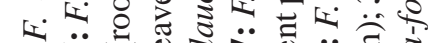
$\ddot{1}$ เ

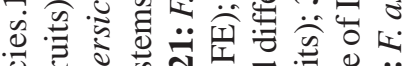

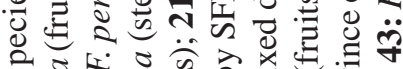
की हा I

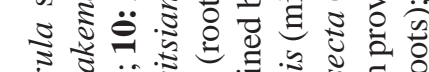
วิ 讧 营

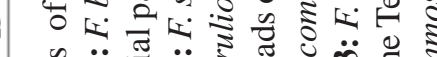

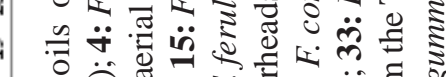

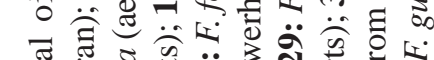
. 접

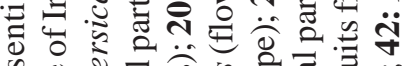

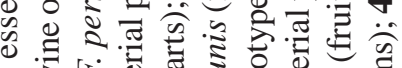

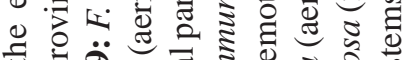
‡ .

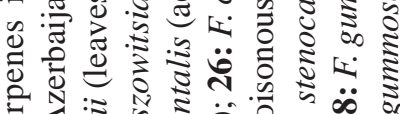
它选

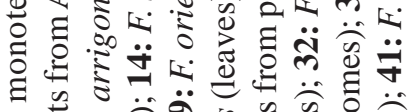

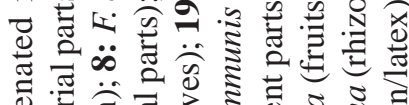

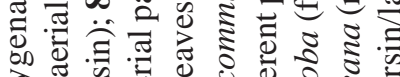

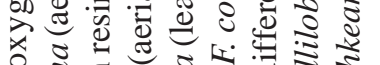

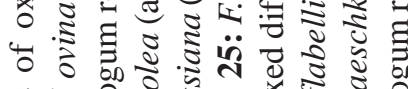

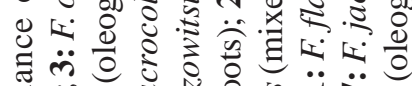

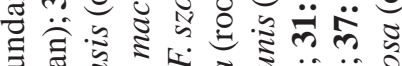

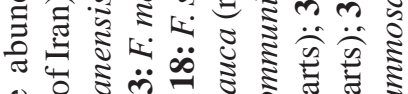

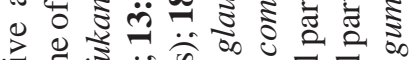

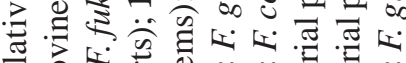

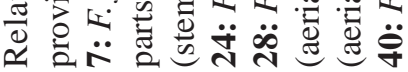
$\stackrel{\nabla 0}{\circ}$ 


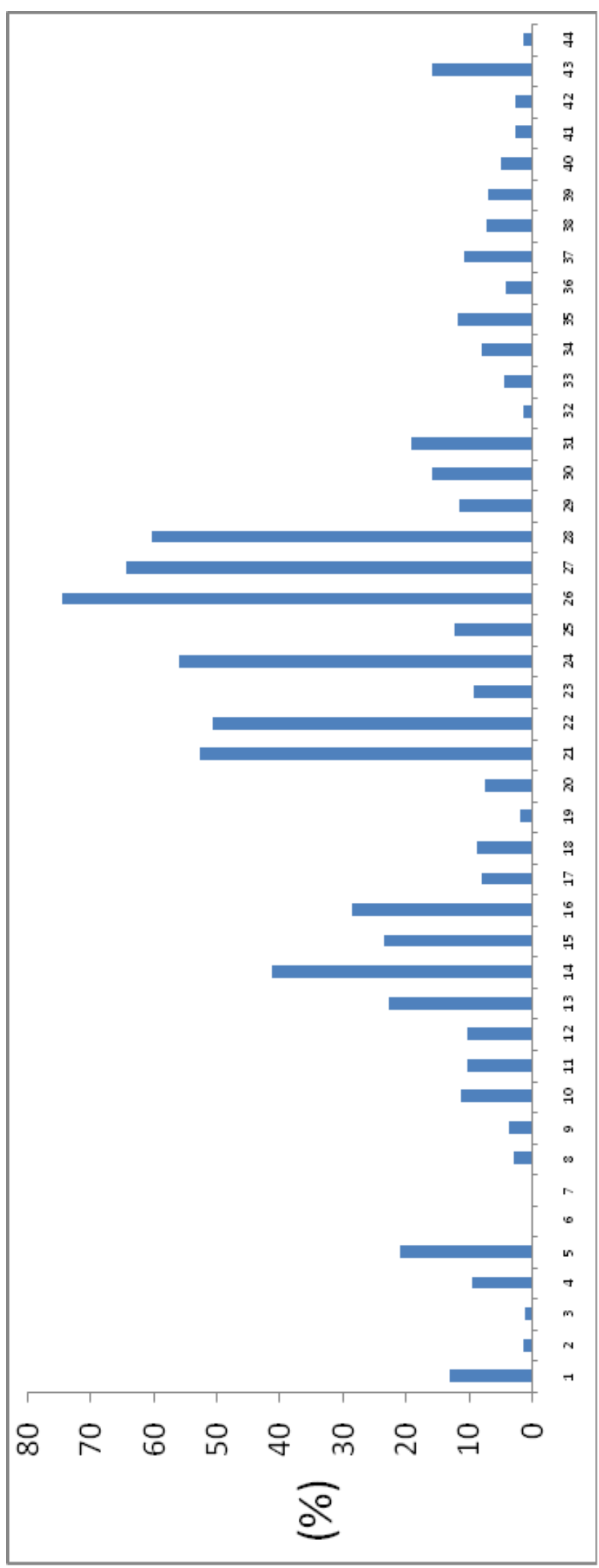

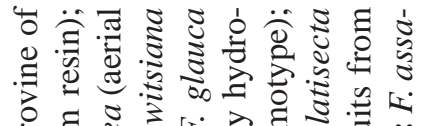

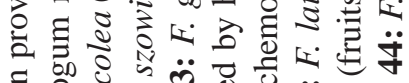
矛完

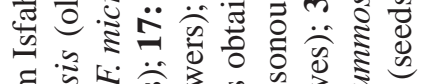
घ:

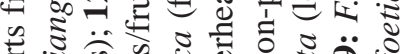

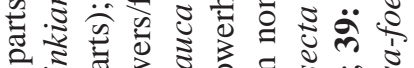

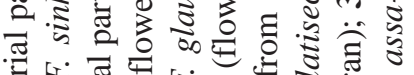

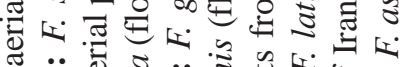

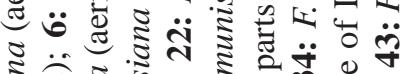

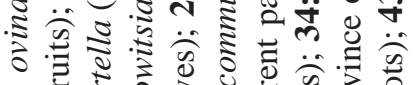

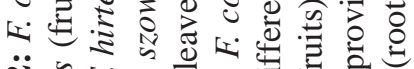
ㄱ.

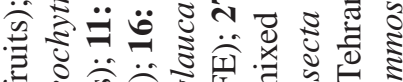

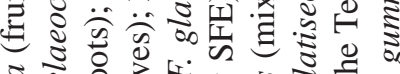

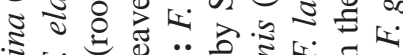

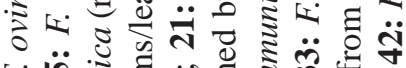
琎

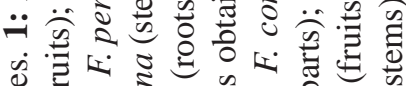

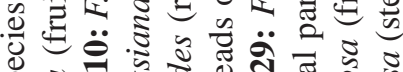
के ह

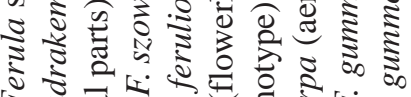

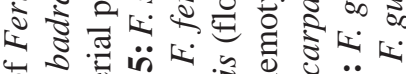

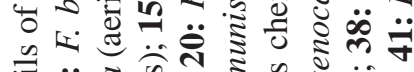

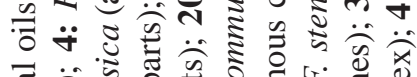

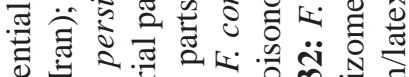
ฮิ

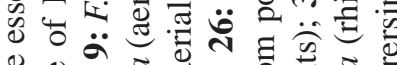

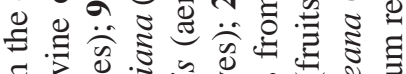

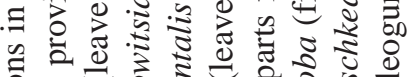

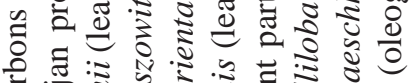
䒕: : : :

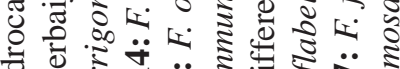

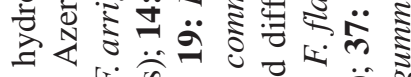

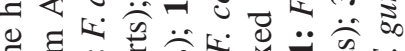

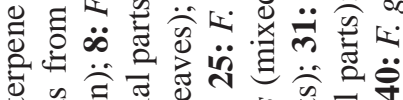

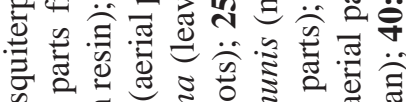
岛.] प्.

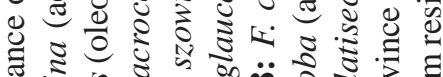

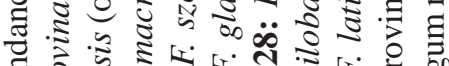

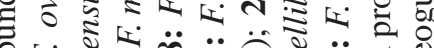
चै

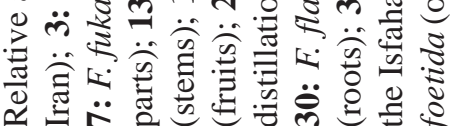
. ำ 

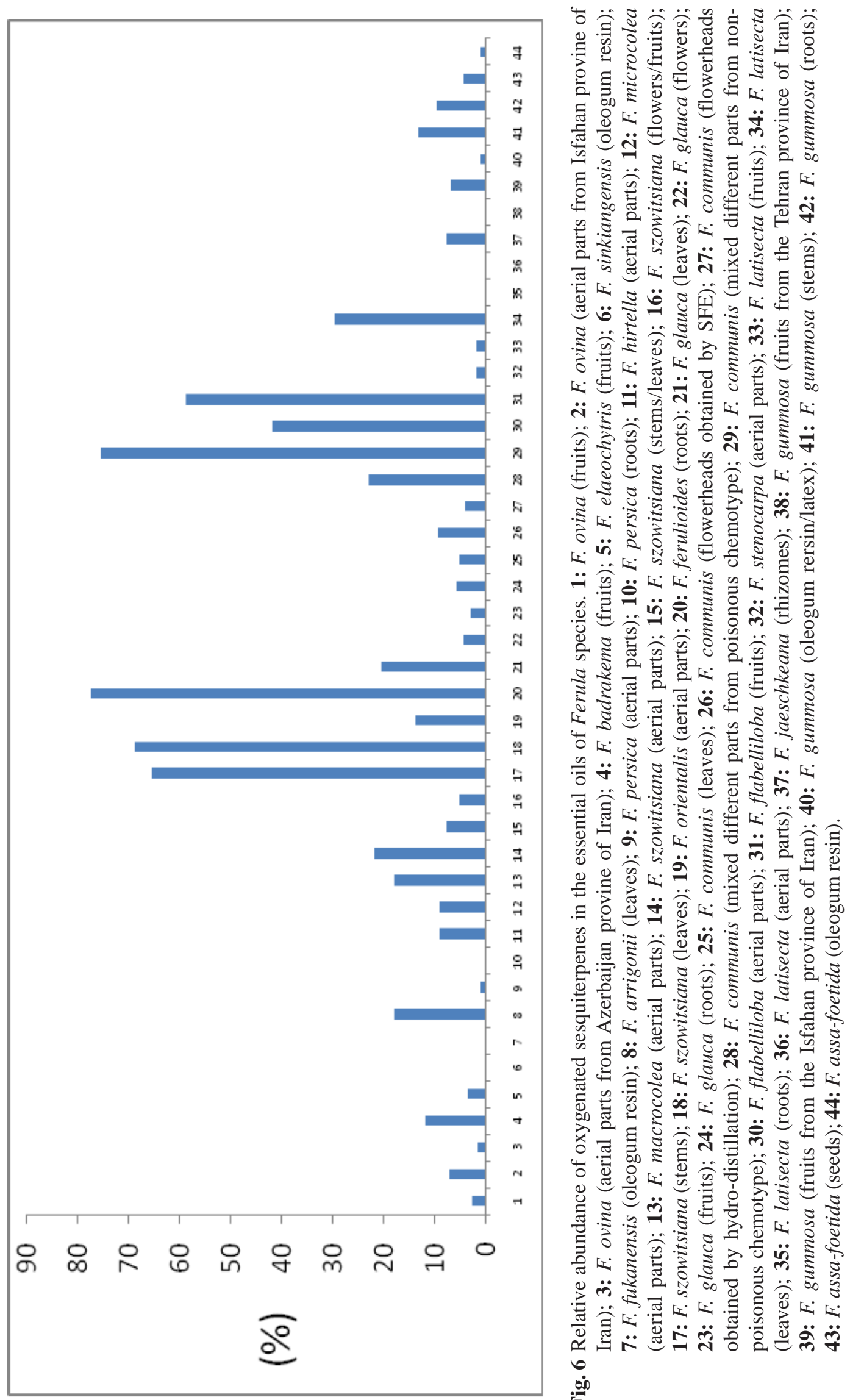


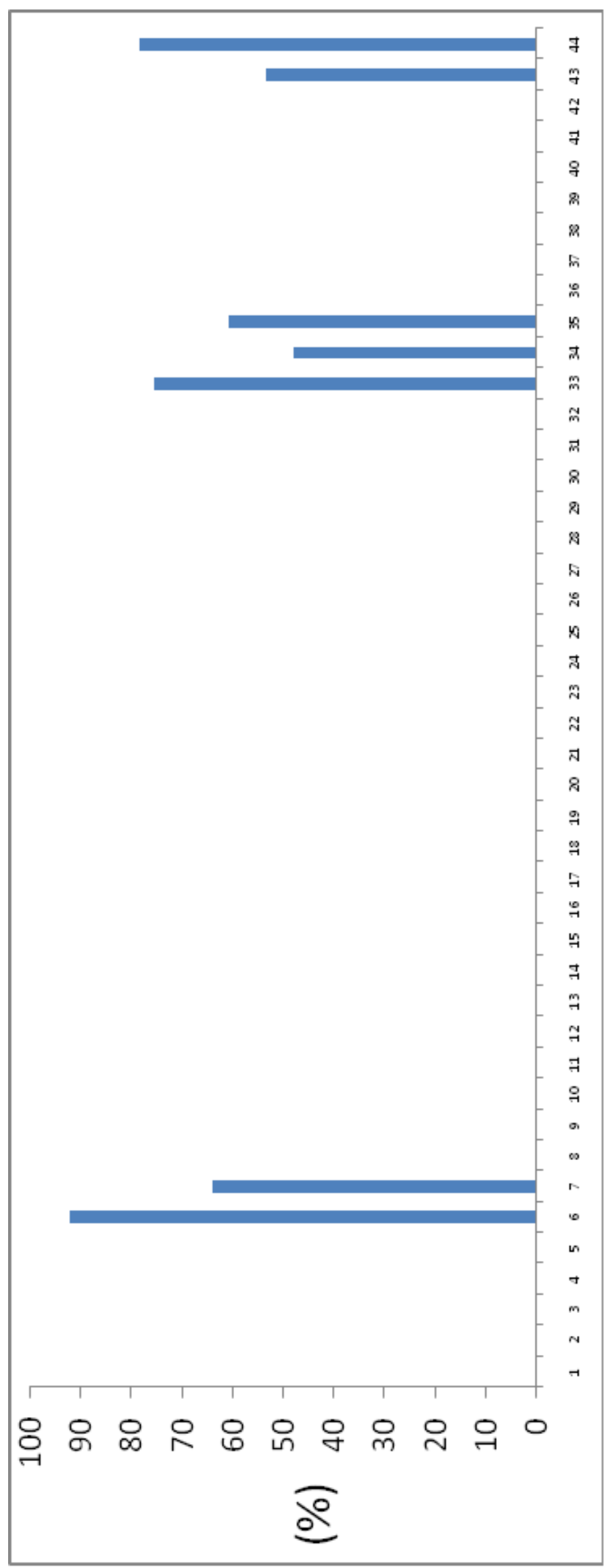

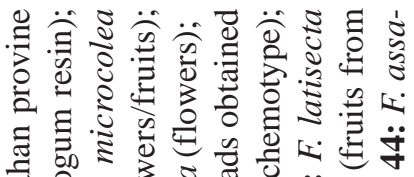
跑 导圷 E

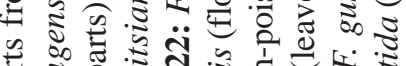

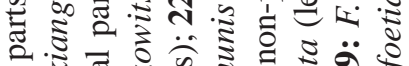

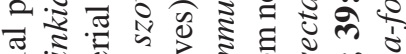

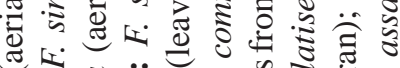

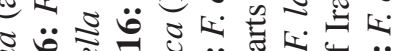

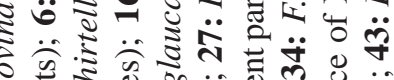
ठ气 :

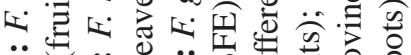

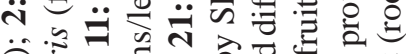
ஸे

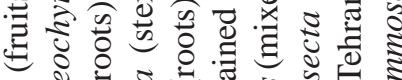

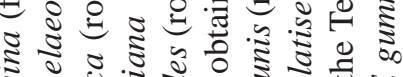

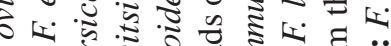

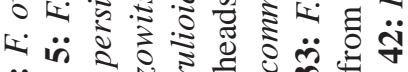

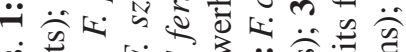

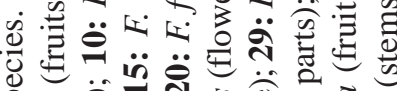

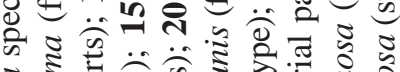

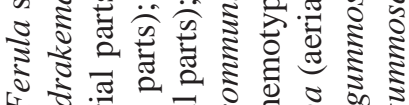

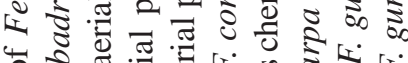

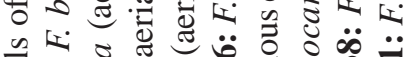

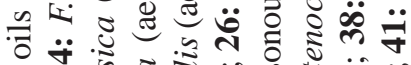
.

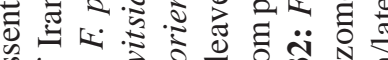
के

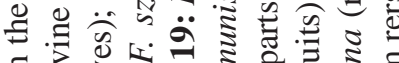

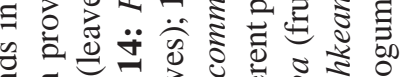

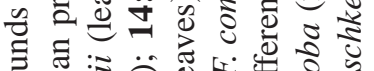
寻: :

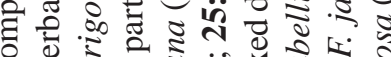

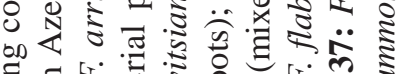

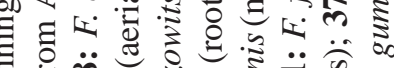

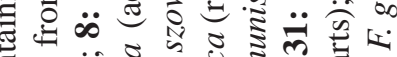

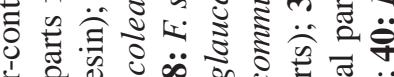

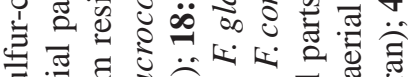

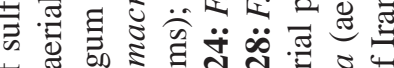

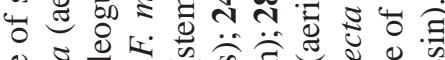

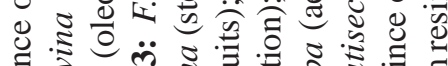

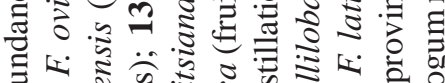

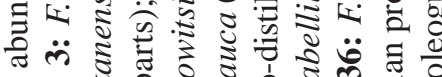
-

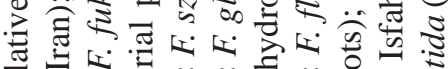

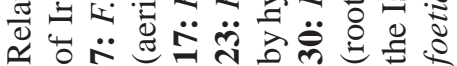

م. 


\section{References}

1. Hedge C, Lamond JM, Rechinger KH, Alava R, Chamberlain DF, Engstrand L, et al. Umbelliferae. In: Rechinger KH, ed. Flora Iranica. Vol. 162. Graz: Akademische Druck- u. Verlagsanstalt; 1987.

2. Singh V, Jain DK. Taxonomy of angiosperms. 6th ed. India:Rastogi Publications; 2007. p. 298.

3. Pimenov MG, Leonov MV. The genera of the Umbelliferae. Kew:Royal Botanic Gardens; 1993.

4. Mozaffarian V. The Family of Umbelliferae in Iran- Keys and Distribution. Tehran:Research Institute of Forests and Rangelands Press; 1983. p. 114-6.

5. Mozaffarian V. A Dictionary of Iranian Plant Names. Tehran:Farhang-e Moaser; 1996. p. 228-30.

6. Murray RDH, Mendez J, Brown SA. The Natural Coumarins. 1st ed. New York:John Wiley \& Sons Inc; 1982. p. 555-9.

7. Iranshahi M, Hassanzadeh-Khayyat M, Sahebkar A, Famili A. Chemical composition of the fruit oil of Ferula flabelliloba. J Essent Oil-Bear Plants. 2008; 11:143-7.

8. Iranshahi M, Kalategi F, Sahebkar A, Sardashti A, Schneider B. New sesquiterpene coumarins from the roots of Ferula flabelliloba. Pharm Biol. 2010; 60:657-2.

9. Motai T, Daikonya A, Kitanaka S. Sesquiterpene Coumarins from Ferula fukanensis and nitric oxide production inhibitory effects. J Nat Prod. 2004; 67: 432-6.

10. Iranshahi M, Shahverdi AR, Mirjani R, Amin GR, Shafiee A. Umbelliprenin from Ferula persica roots inhibits the red pigment production in Serratia marcescens. Z Naturforsch. 2004; 59 c:506-8.

11. Iranshahi M, Arfa P, Ramezani M, Jaafari MR, Sadeghian H, Bassarello C, et al. Sesquiterpene coumarins from Ferula szowitsiana and in vitro antileishmanial activity of 7-prenyloxycoumarins against promastigotes. Phytochemistry. 2007; 68: 554-61.

12. Iranshahi M, Kalategi F, Rezaee R, Shahverdi AR, Ito C, Furukawa H, et al. Cancer chemopreventive activity of terpenoid coumarins from Ferula species. Planta Med. 2008; 74:147-50.

13. Kogure K, Yamauchi I, Tokumura A, Kondou K, Tanaka $\mathrm{N}$, Takaishi Y, et al. Novel antioxidants isolated from plants of the genera Ferula, Inula, Prangos and Rheum collected in Uzbekistan. Phytomedicine. 2004; 11: 645-51.

14. Tamemoto K, Takais Y, Chen B, Kawazoe K, Shibata H, Higuti T, et al. Sesquiterpenoids from the fruits of Ferula kuhistanica and antibacterial activity of the constituents of F. kuhistanica. Phytochemistry. 2001;
58:763-7.

15. Iranshahi M, Rezaee R, Sahebkar A, Bassarello C, Piacente S, Pizza C. Sesquiterpene coumarins from the fruits of Ferula badrakema. Pharm Biol. 2009; 47: 344-7.

16. Iranshahi M, Sahebkar A, Hosseini ST, Takasaki M, Konoshima T, Tokuda H. Cancer chemopreventive activity of diversin from Ferula diversivittata in vitro and in vivo. Phytomedicine. 2010; 17:269-73.

17. Iranshahi M, Sahebkar A, Takasaki M, Konoshima T, Tokuda H. Cancer chemopreventive activity of the prenylated coumarin, umbelliprenin, in vivo. Eur J Cancer Prev. 2009; 18:412-5.

18. Rajanikanth B, Ravindranath B, Shankaranarayana ML. Volatile Polysulphides of Assafoetida. Phytochemistry. 1984; 23:899-900.

19. Al-said MS, Abdel Sattar E, El-Feraly F, Nahrstedt A, Coen M. New Sulfides from Ferula rutabensis. Int J Pharmacog. 1996; 34:189-93.

20. Iranshahi M, Amin G, Amini M, Shafiee A. Sulfur containing derivatives from Ferula persica var. latisecta. Phytochemistry. 2003; 63:965-6.

21. Iranshahi M, Amin G, Salehi-Sourmaghi MH, Shafiee A, Hadjiakhoondi A. Sulphur-containing compounds in the essential oil of the root of Ferula persica Willd. var. persica. Flav Fragr J. 2006; 21:260-1.

22. Zhi-da M, Qi-fi M, Mizuno M, Tanaka T, Iinuma M. Polysulfanes in the volatile oil of Ferula species. Planta Med. 1987; 53:300-2.

23. Iranshahi M, Yazdi MC, Hassanzadeh-Khayyat M, Sahebkar A. Sulfur containing compounds in the volatile oil of Ferula latisecta Rech. f. \& Aell. Leaves. J Essent Oil-Bear Plants. 2009; 12:64-8.

24. Sahebkar A, Hassanzadeh-Khayyat M, Iranshahi M. Qualitative analysis of the hydro-distilled essential oil of Ferula latisecta Rech. f. \& Aell. Roots from Iran. J Essent Oil-Bear Plants. 2010; 13:340-6.

25. Zargari A. Medicinal Plants (in Persian). Vol. 2. Tehran: Tehran University Publications; 1996. p. 592-602.

26. Eigner D, Scholz D. Das zauberb chlein der gyani dolma. Pharm Unserer Zeit. 1990; 19:141-52.

27. Afifi FU, Abu-Irmaileh B. Herbal medicine in Jordan with special emphasis on less commonly used medicinal herbs. J Ethnopharmacol. 2000; 72:101-10.

28. Plants for a Future [online] Available from http:// www.pfaf.org/index.php [Accessed February 02, 2009]

29. Asili J, Sahebkar A, Fazly Bazzaz BS, Sharifi S, Iranshahi M. Identification of essential oil components of Ferula badrakema fruits by GC-MS and13C-NMR methods and evaluation of its antimicrobial activity. J Essent 
Oil-Bear Plants. 2009; 12:7-15.

30. Maggi F, Cecchini C, Cresci A, Coman MM, Tirillini B, Sagratini G, et al. Chemical composition and antimicrobial activity of the essential oil from Ferula glauca L. (F. communis L. subsp. glauca) growing in Marche (central Italy). Fitoterapia. 2009; 80:68-72.

31. Iranshahi M, Fata A, Emami B, Shahri BMJ, Fazly Bazzaz BS. In vitro antifungal activity of polysulfidesrich essential oil of Ferula latisecta fruits against human pathogenic dermatophytes. Nat Prod Commun. 2008; 3:1543-6.

32. Kartal N, Sokmen M, Tepe B, Daferera D, Polissiou M, Sokmen A. Investigation of the antioxidant properties of Ferula orientalis $L$. using a suitable extraction procedure. Food Chem. 2007; 100:584-9.

33. Appendino G, Mercalli E, Fuzzati N, Arnoldi L, Stavri M, Gibbons S, et al. Antimycobacterial coumarins from the Sardinian giant fennel (Ferula communis). J Nat Prod. 2004; 67:2108-10.

34. Mossa JS, El-Feraly FS, Muhammad I. Antimycobacterial constituents from Juniperus procera, Ferula communis and Plumbago zeylanica and their in vitro synergistic activity with isonicotinic acid hydrazide. Phytother Res. 2004; 18:934-7.

35. Mandegary A, Sayyah M, Reza Heidari M. Antinociceptive and anti-inflammatory activity of the seed and root extracts of Ferula gummosa Boiss in mice and rats. Daru. 2004; 12:58-62.

36. Fatehi M, Farifteh F, Fatehi-Hassanabad Z. Antispasmodic and hypotensive effects of Ferula asafoetida gum extract. J Ethnopharmacol. 2004; 91: 321-4.

37. Sayyah M, Mandgary A. Anticonvulsant effect of Ferula gummosa root extract against experimental seizures. Iran Biomed J. 2003; 7:139-43.

38. Sayyah M, Mandgary A, Kamalinejad M. Evaluation of the anticonvulsant activity of the seed acetone extract of Ferula gummosa Boiss. against seizures induced by pentylenetetrazole and electroconvulsive shock in mice. J Ethnopharmacol. 2002; 82:105-9.

39. Dehghan G, Shafiee A, Ghahremani MH, Ardestani SK, Abdollahi M. Antioxidant potential of various extracts from Ferula szovitsiana in relation to their phenolic content. Pharm Biol. 2007; 45:691-9.

40. Bakkali F, Averbeck S, Averbeck D, Idaomar M. Biological effects of essential oils - a review. Food Chem Toxicol. 2008; 46:446-75.

41. Perry NSL, Bollen C, Perry EK, Ballard C. Salvia for dementia therapy: review of pharmacological activity and pilot tolerability clinical trial. Pharm Biochem
Behav. 2003; 75:651-9.

42. Silva J, Abebe W, Sousa SM, Duarte VG, Machado MIL, Matos FJA. Analgesic and antiinflammatory effects of essential oils of eucalyptus. J Ethnopharmacol. 2003; 89:277-83.

43. Hajhashemi V, Ghannadi A, Sharif B. Anti-inflammatory and analgesic properties of the leaf extracts and essential oil of Lavandula angustifolia Mill. J Ethnopharmacol. 2003; 89:67-71.

44. Iranshahi M, Hassanzadeh-Khayat M, Bazzaz BSF, Sabeti Z, Enayati F. High content of polysulphides in the volatile oil of Ferula latisecta rech. F. et Aell. fruits and antimicrobial activity of the oil. J Essent Oil Res. 2008; 20:183-5.

45. Habibi Z, Salehi P, Yousefi M, Hejazi Y, Laleh A, Mozaffarian V, et al. Chemical composition and antimicrobial activity of the essential oils of Ferula latisecta and Mozaffariania insignis from Iran. Chem Nat Comp. 2006; 42:689-92.

46. Ghasemi Y, Faridi P, Mehregan I, Mohagheghzadeh A. Ferula gummosa fruits: an aromatic antimicrobial agent. Chem Nat Comp. 2005; 41:311-4.

47. Eftekhar F, Yousefzadi M, Borhani K. Antibacterial activity of the essential oil from Ferula gummosa seed. Fitoterapia. 2004; 75:758-9.

48. Ozek G, OzekT, Iscan G, Baser KHC, Duran A, Hamzaoglu E. Composition and antimicrobial activity of the oils of Ferula szowitsiana DC. from Turkey. J Essent Oil Res. 2008; 20:186-90.

49. Dehghan G, Solaimanian R, Shahverdi AR, Amin G, Abdollahi M, Shafiee A. Chemical composition and antimicrobial activity of essential oil of Ferula szovitsiana DC. Flav Fragr J. 2007; 22:224-7.

50. Rahman MU, Gul S. Antimicrobial activities of Ferula assafoetida oil. Part I. Inhibition of sexual reproduction stages of foodborne molds. J Chem Soc Pakistan. 2005; 27:423-8.

51. Sayyah M, Kamalinejad M, Hidage RB, Rustaiyan A. Antiepileptic potential and composition of the fruit essential oil of Ferula gummosa Boiss. Iran Biomed J. 2001; 5:15-16.

52. Sadraei H, Asghari GR, Hajhashemi V, Kolagar A, Ebrahimi M. Spasmolytic activity of essential oil and various extracts of Ferula gummosa Boiss. on ileum contractions. Phytomedicine. 2001; 8:370-6.

53. El-Thaher TS, Matalka KZ, Taha HA, Badwan AA. Ferula harmonis 'zallouh' and enhancing erectile function in rats: Efficacy and toxicity study. Int J Impotence Res. 2001; 13:247-51.

54. Duke JA, Beckstrom SM. Handbook of Medicinal 
Mints, Phytochemicals, and Biological Activities. Florida:CRC Press; 1996. p. 379-83.

55. Kim S, Kubec R, Musah RA. Antibacterial and antifungal activity of sulfur-containing compounds from Petiveria alliacea L. J Ethnopharmcol. 2006; 104: 188-92.

56. Benevides PJC, Young MCM, Giesbrecht AM, Roque NF, Da Bolzani VS. Antifungal polysulphides from Petiveria alliacea L. Phytochemistry. 2001; 57:743-7.

57. Tasaka A, Tamura N, Matsushita Y, Hayashi R, Okonogi K, Itoh K. Optically active antifungal azoles. II. Synthesis and antifungal activity of polysulfide derivatives of (2R,3R)-2-(2,4-difluorophenyl)-3-
mercapto-1-(1H-1,2,4-triazol-1-yl)-2-butanol. Chem Pharm Bull. 1993; 41;1043-8.

58. Kubota K, Saori O, Kobayashi A. Identification and antimicrobial activity of the volatile flavor constituents from Scorodocarpus borneensis Becc. Biosci Biotech Bioch. 1994; 58:644-6.

59. Shahwan MJ, Al-Qirim TM, Daradka H. Effects of Ballota undulate on blood biochemical parameters and insulin in albino rat. Asian Biomed. 2009; 3:171-5.

60. Jeyyabalan S, Palayan M. Antihyperglycemic and antidiabetic activity of leaves extracts of Sapindus emarginatus Vahal. Asian Biomed. 2009; 3:313-7. 